\title{
Experimental Investigation of Inter- element Isolation in a Medical Array Transducer at Various Manufacturing Stages
}

\author{
Franco Marinozzi', Fabiano Bini', and Andrea Grandoni²
}

\begin{abstract}
This work presents the experimental investigation of vibration maps of a linear array transducer with 192 piezoelements by means of a laser Doppler vibrometer at various manufacturing finishing steps in air and in water. Over the years, many researchers have investigated cross-coupling in fabricated prototypes but not in arrays at various manufacturing stages. Only the central element of the array was driven at its working frequency of $5 \mathrm{MHz}$. The experimental results showed that the contributions of cross-coupling depend on the elements of the acoustic stack: Lead Zirconate Titanate (PZT), kerf, filler, matching layer, and lens. The oscillation amplitudes spanned from $(6 \pm 38 \%) \mathrm{nm}$ to $(110 \pm 40 \%) \mathrm{nm}$ when the energized element was tested in air and from $(6 \pm 57 \%) \mathrm{nm}$ to $(80 \pm 67 \%) \mathrm{nm}$ when measurements were obtained under water. The best interelement isolation of $-22 \mathrm{~dB}$ was measured in air after cutting the kerfs, whereas the poorest isolation was $-2 \mathrm{~dB}$ under water with an acoustic lens (complete acoustic stack). The vibration pattern in water showed a higher standard deviation on the displacement measurements than the one obtained in air, due to the influence of acousto-optic interactions. The amount increased to $30 \%$ in water, as estimated by a comparison with the measurements in air. This work describes a valuable method for manufacturers to investigate the correspondence between the manufacturing process and the quantitative evaluations of the resulting effects.
\end{abstract}

\section{Keywords}

array transducers, inter-element cross-coupling, laser Doppler vibrometry, manufacturing process

\section{Introduction}

Ultrasound medical imaging has become increasingly more demanding with transducer performance evaluation and optimization. The characterization of ultrasound arrays for medical imaging, in-house process development, and investment in state-of-the-art fabrication equipment are key issues for teams of scientists, engineers, and technicians.

\footnotetext{
'Department of Mechanical and Aerospace Engineering, Sapienza University of Rome, Rome, Italy ${ }^{2}$ Transducer Manufacturing Engineer, Industrial Engineering Group ESAOTE S.p.A, Florence, Italy

\section{Corresponding Author:}

Franco Marinozzi, Department of Mechanical and Aerospace Engineering, Sapienza University of Rome, Eudossiana I8, 00184 Rome, Italy.

Email: franco.marinozzi@uniromal.it
} 
Real-time imaging performance deteriorates with inter-element cross-coupling, which decreases the sensitivity and angular directivity, and increases the acoustic noise level. Many researchers have investigated this phenomenon with various prototypes ${ }^{1-8}$ over the years.

Inter-element coupling has been identified to be the major problem in manufacturing electronic multi-element array transducers. Evaluation of the performance of array transducers is usually carried out by measuring impulse responses, radiation patterns, and cross-coupling between neighboring elements. The inter-element isolation quantifies the unwanted excitation of elements adjacent to a single, electrically driven, element. Ideally, when an electrical impulse is applied to a piezoelement, the adjacent ones should not be activated. This condition is hardly ever achievable. In fact, all the various components of an acoustic stack are mechanically coupled via filling materials, matching layers, the acoustic lens, the backing, and the probe housing.

Another cause of mechanical coupling is due to the excitation of spurious vibration modes ${ }^{9}$ within the array. All these simultaneous factors can hinder suitable mechanical isolation.

To analyze the complex mechanical behavior of an array transducer, it is mandatory to measure the displacements of the array elements. Once the vibration map of the array surface has been obtained, the manufacturer can modify the manufacturing processes and optimize the electromechanical conversion efficiency to improve the image quality.

According to the authors' knowledge, different techniques have been adopted to measure the level of inter-element isolation. One of the most accepted is the analysis of the electrical impedance of adjacent elements.

In particular, the influence of the filling material is analyzed with respect to the piezoelement and the matching layer via the electrical impedance. ${ }^{10,11}$ The latter references gives evidence for how hard it is to achieve the inter-element isolation because of the complex electromechanical couplings that unavoidably arise during the manufacturing. Lamberti et al. ${ }^{12}$ have measured the vibration amplitude of ad hoc arrays, without matching layers, built by using the classical dice and fill technique. They have investigated the influence of three different filling materials on the alteration of the radiation patterns. Felix et al. ${ }^{13,14}$ have adopted the Laser interferometery, instead of more traditional techniques based on an hydrophone and a network analyzer. Dias ${ }^{15}$ has experimentally analyzed the presence of low frequency $(0.83 / 0.97 \mathrm{MHz})$ acoustic modes, which couple different elements via surface wave propagation on the backing surface. The propagation of mechanical waves through the backing layer is relevant only for materials having a relatively low mechanical absorption (for instance, the attenuation of the composite Spurr Epoxy and Alumina is $3.5 \mathrm{~dB} \cdot \mathrm{cm}^{-1}$ at $5 \mathrm{MHz}$ ). In addition, experimental results have shown that cuts in the backing and matching layers reduce the cross-talk effect. However, different vibration patterns, such as Lamb modes, ${ }^{16}$ naturally arise in the propagation along the matching layers. Moreover, it is difficult to simulate the behavior of a filling material because its real acoustic properties depend on several unpredictable factors, such as the doping and/or the curing process during the manufacturing. The critical parameter of the production process is given by the polymerization of the filling material and adhesive. Moreover, the polymerization process strongly depends on environmental factors such as temperature, heating/cooling rates, and humidity, and thus the real mechanical behavior of the filling material cannot be easily simulated, regardless of the powerful analysis technique based on the Finite Element Method. ${ }^{17}$ This implies that some techniques have been adopted to reduce inter-element cross-coupling; for example, sub-dicing, may not yield the expected results. Therefore, the desired uncoupling is achievable only theoretically. It must be noted that the cited studies of the inter-element cross-coupling were performed only on prototypal array transducers, which are, to a large extent different from the commercialized ones.

The models commonly used to simulate the mechanical and electrical behavior of piezoelectric transducers generally introduce simplifying assumptions that are often invalid for actual designs. For example, the most accepted models, such as Mason ${ }^{18}$ and Krimholtz et al. ${ }^{19}$ are only one dimensional (1D). 
The aim of this study was to perform experimental investigation ${ }^{20-22}$ of the vibration amplitude of ultrasound arrays harmonically excited at the working frequency by means of a laser Doppler vibrometer (LDV). In particular, we focused our work on inter-element cross-coupling of the acoustic stack at various manufacturing finishing stages. Similar measurements have not been reported in the literature.

The measured vibration maps confirmed the complexity of the phenomenon and represent the starting point for further theoretical and experimental analysis of the oscillatory behavior of commercialized arrays transducers.

\section{Experimental Set-Up}

To take into account inter-element cross-coupling, it is necessary to measure the amplitudes of the vibrations of a single energized piezoelement. ${ }^{15}$ The vibration amplitudes have been measured by means of an experimental set-up, shown in Figure 1a, both in air and placed in a water tank. This latter setup was adopted, according to Felix et al. ${ }^{13}$ (Figure 1b), because the acoustic impedance of water (1.48 Mrayl) is similar to that of biological soft tissues (1.68 Mrayl).

The probing beam of the LDV was transmitted to the transducer through a transparent and plane-parallel fused silica plate. To reduce undesired vibrations, the tank and the LDV were mounted on an optical bench.

Measurements were carried out on a linear array transducer, with a working frequency of 5 $\mathrm{MHz}$ and 192 piezoelements. Typical nominal values were length $(l=6 \mathrm{~mm})$, thickness $(t=0.3$ $\mathrm{mm})$, width $(w=0.245 \mathrm{~mm})$, and array pitch $(d=0.270 \mathrm{~mm})$. The kerf width was $0.025 \mathrm{~mm}$ (Figure 1b). For measurements in the air, the distance between the laser sensor head and the transducer should be $175 \mathrm{~mm}$, so that the laser beam diameter (waist size) is equal to $10 \mu \mathrm{m}$. The LDV acquires an average of 32 traces, that is, $2 \mu$ s for each point. The interval between two consecutive acquisitions was approximately $3 \mathrm{~s}$. This choice was consistent with the acquisition and processing time, as well as the positioning of the transducers between two distinct points. All the data were stored and processed later to compute the peak-to-peak amplitude of the Fourier transform for a working frequency of $5 \mathrm{MHz}$ at the scanning point.

The peak-to-peak amplitude of the vibrations ${ }^{23,24}$ was measured via LDV (POLYTEC). This instrument has two main elements: (a) an optical sensor head OFV 353, class II, Helium-Neon laser source $\lambda=633 \mathrm{~nm}$, output power from 0.8 to $1 \mathrm{~mW}$, and (b) the signal processor OFV 27002, from $25 \mathrm{kHz}$ to $20 \mathrm{MHz}$ bandwidth, $50 \mathrm{~nm} \cdot \mathrm{V}^{-1}$ sensitivity, and $10^{-3} \AA \cdot \mathrm{Hz}^{-1 / 2}$ resolution at $100 \%$ reflectivity in air.

The experimental bench, shown in Figure 1a, consisted of a function generator (AGILENT TECHNOLOGIES 33250A), which was synchronized via an external trigger signal emitted by an 8-bit digital oscilloscope (YOKOGAWA DL 1540) with sampling rates of up to $200 \mathrm{MS} / \mathrm{s}$ and a $150 \mathrm{MHz}$ bandwidth.

The signal output of the signal processor OFV 2700-2 was the signal input to the spectrum/ network analyzer HP4195A and the oscilloscope, which was controlled by a personal computer via a GPIB interface (PCI/GPIB NATIONAL INSTRUMENTS) and an IEEE 488 bus. Data were acquired via ad hoc software. The sensor head laser was translated by two micro positioning stages, of the M-400 DG series (PHYSIK INSTRUMENTE; bi-directional repeatability $\pm 2 \mu \mathrm{m}$ ) that were assembled orthogonally to scan the surface in the $x-y$ plane of the transducer. The micro positioning stages were connected via a RS232 serial cable to a C-844 DC motor controller (PHYSIK INSTRUMENTE) that was controlled via a data acquisition board (PCI/GPIB NATIONAL INSTRUMENTS) and the IEEE 488 bus.

Each piezoelement was sub-diced in two sub-elements, which split the whole part without interrupting the signal electrode. Only one piezoelement (Figures $2 \mathrm{a}$ and $2 \mathrm{~b}$ ), the number 96, was activated, because it was the central element of the transducer. Therefore, the excited piezoelements for 


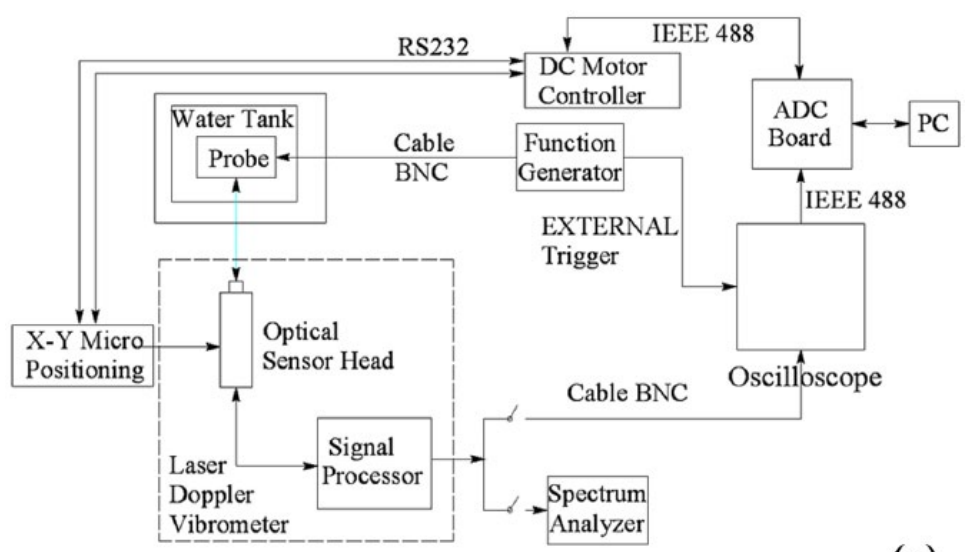

(a)

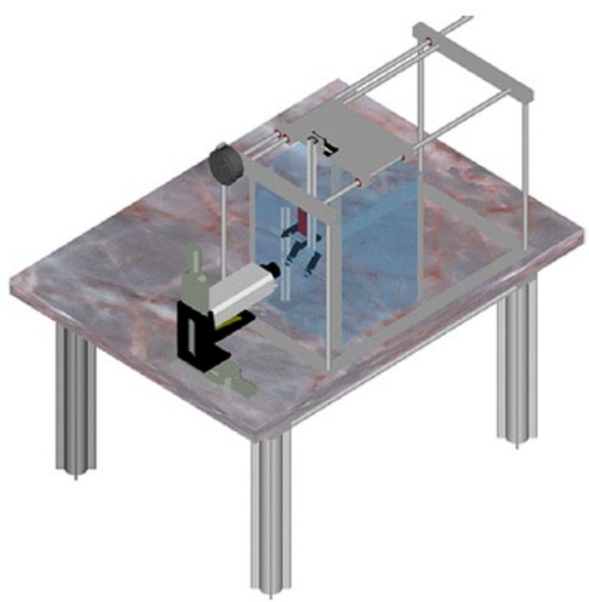

(b)

Figure I. (a) Block diagram of the experimental set-up; (b) 3D view of the LDV for tests under the water tank. 3D = three dimensional; $L D V=$ laser Doppler vibrometer; $A D C=$ Analog to Digital Converter; BNC = Bayonet Neill-Concelman connector.

electromechanical coupling in air and under water are given by the sub-element $(95 r)$, to the right of the element 95, and the sub-element (97l), to the left of the piezoelement 97.

The piezoelement was activated by a function generator with 50 cycles of a $5 \mathrm{MHz}$ sinusoidal burst, $10 \mathrm{~V}_{\mathrm{PP}}$ amplitude, and a repetition time of approximately $120 \mu \mathrm{s}$.

The commercialized transducers that were used were at different finishing steps: (a) backing and PZT with the kerf; (b) backing and PZT, matching layers 1 and 2, and the kerf; (c) backing and PZT, matching layers 1 and 2, and the filled kerf; (d) backing and PZT, matching layers 1 and 2 , the filled kerf, and the acoustic lens; and (e) probe completed with the cable and the socket.

\section{Results}

In Figure 3a, we show the mean values of the displacements with the corresponding percentage standard deviation (Figure $3 \mathrm{~b})$. The oscillation amplitudes spanned from $(6 \pm 38 \%) \mathrm{nm}$ to $(110 \pm$ $40 \%) \mathrm{nm}$ when the energized element was tested in air and from $(6 \pm 57 \%) \mathrm{nm}$ to $(80 \pm 67 \%) \mathrm{nm}$ 

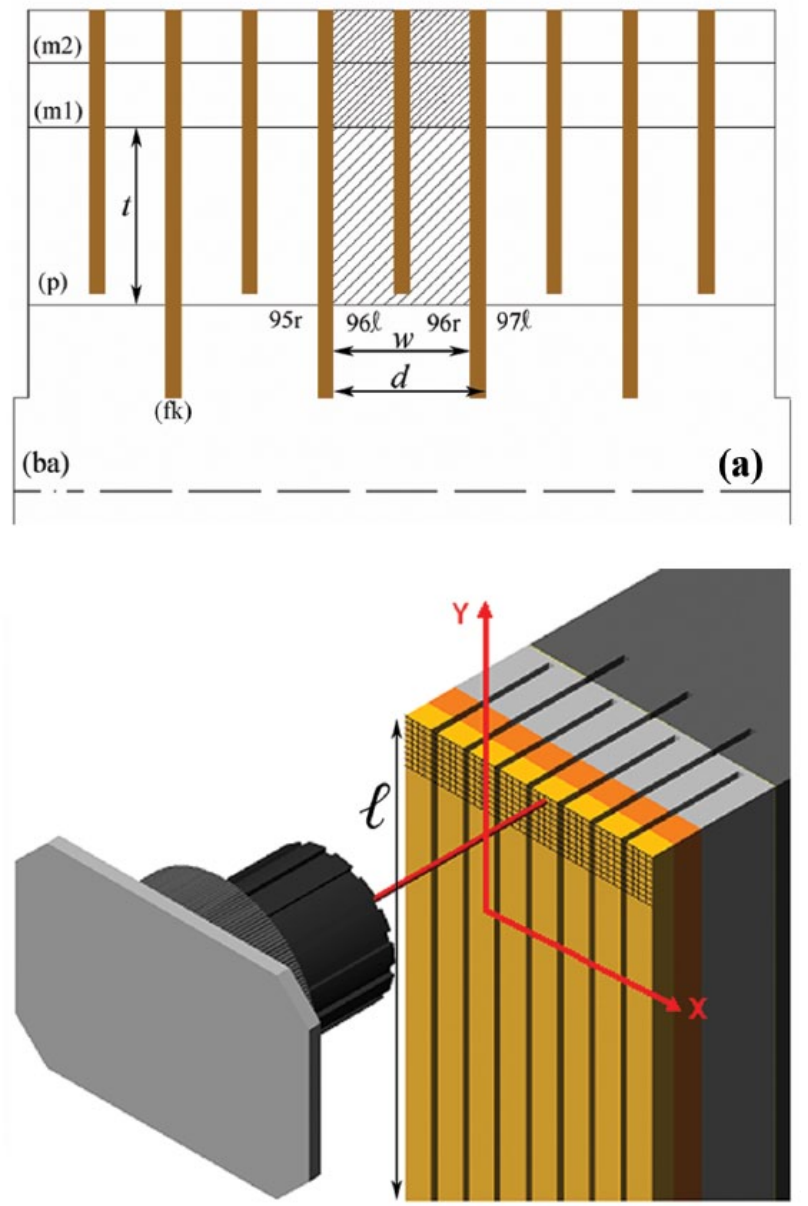

(b)

Figure 2. (a) Sketch of excited and neighboring elements: (ba) backing, ( $p)$ PZT, (m I) matching layer I, (m2) matching layer 2, (fk) filled kerf, thickness $(t)=0.3 \mathrm{~mm}$, width $(w)=0.245 \mathrm{~mm}$, array pitch $(d)=$ $0.270 \mathrm{~mm}$. (b) $3 \mathrm{D}$ view of scanning; length $(l)=6 \mathrm{~mm}$. 3D $=$ three dimensional.

when the measurements were performed in water. The oscillation amplitudes of the neighboring elements spanned from $(4 \pm 48 \%) \mathrm{nm}$ to $(18 \pm 42 \%) \mathrm{nm}$ and from $(5 \pm 62 \%) \mathrm{nm}$ to $(40 \pm 70 \%)$ $\mathrm{nm}$ in air and water, respectively.

The best inter-element isolation (Figure 4) was obtained in air for a transducer with kerf (Step 2 ), and attenuation values were approximately equal to $-22 \mathrm{~dB}$. Conversely, the smallest isolation obtained was from a transducer under water with acoustic lens (Step 4) equal to $-2 \mathrm{~dB}$.

With these mean inter-element isolation values, it is possible to predict the alteration on the directivity of the single element, according to Kino and DeSilets, ${ }^{2}$ and we report the following equations for the directivity function with $(F)$ and without $\left(F_{0}\right)$ cross-coupling:

$$
F_{0}(\theta)=A \sin \left[k_{p} w \sin \left(\frac{\theta}{2}\right)\right],
$$




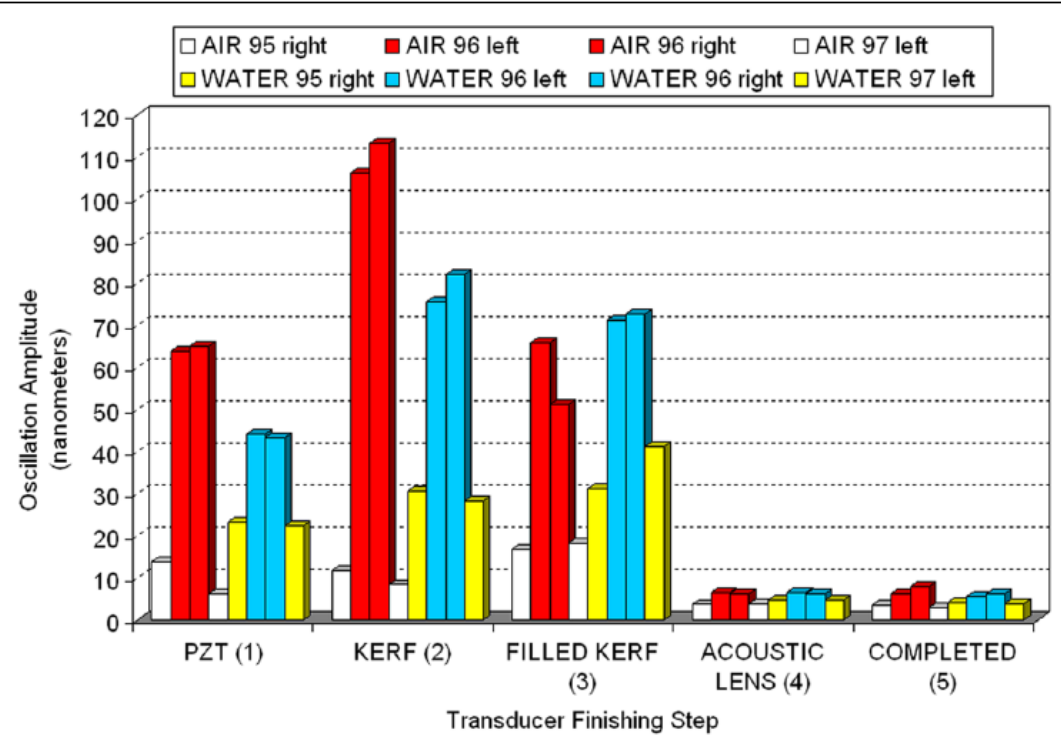

(a)

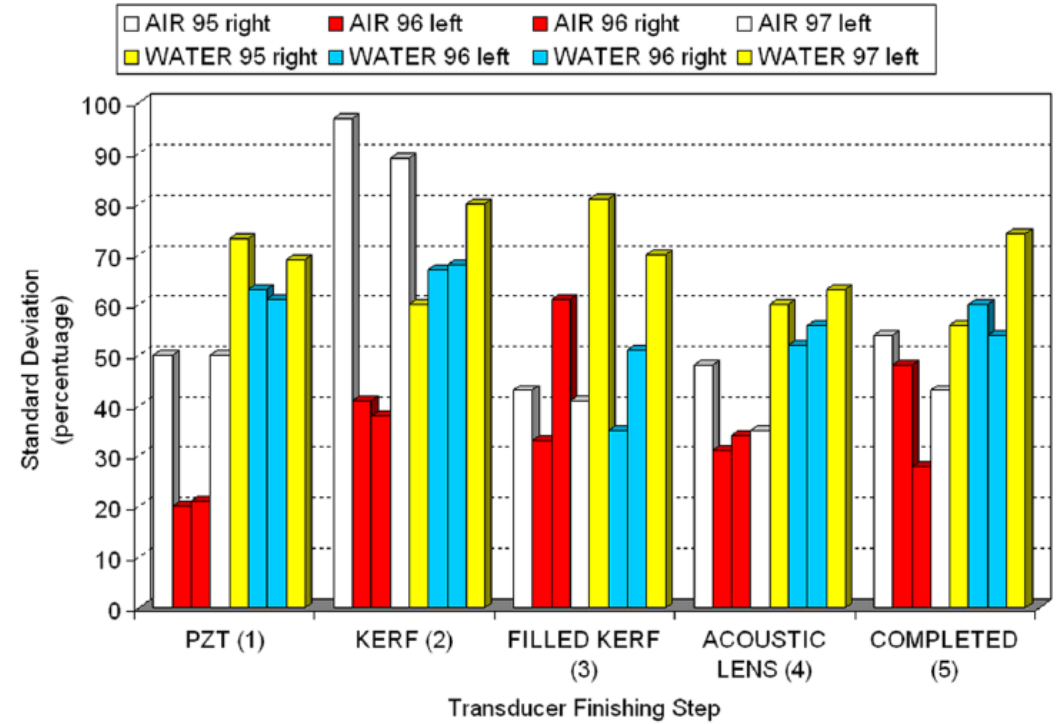

(b)

Figure 3. (a) Mean values in air and under water; (b) standard deviation values in air and under water.

$$
F(\theta)=F_{0}(\theta)\left[1+2 \sum_{n=0}^{N} \alpha^{n} \mathrm{e}^{j n \xi} \cos \left(n k_{p} d \sin \theta\right)\right]
$$

Referring to Equations (1) and (2), in Figure 5, it is evident that the main lobe narrows because of a strong inter-element coupling; that is, $\alpha=-2 \mathrm{~dB}=0.794$, as measured in this work. Given the relatively low power loss within the filler, we assumed a phase lag $\xi=0^{\circ}$ between the energized and the coupled elements. 


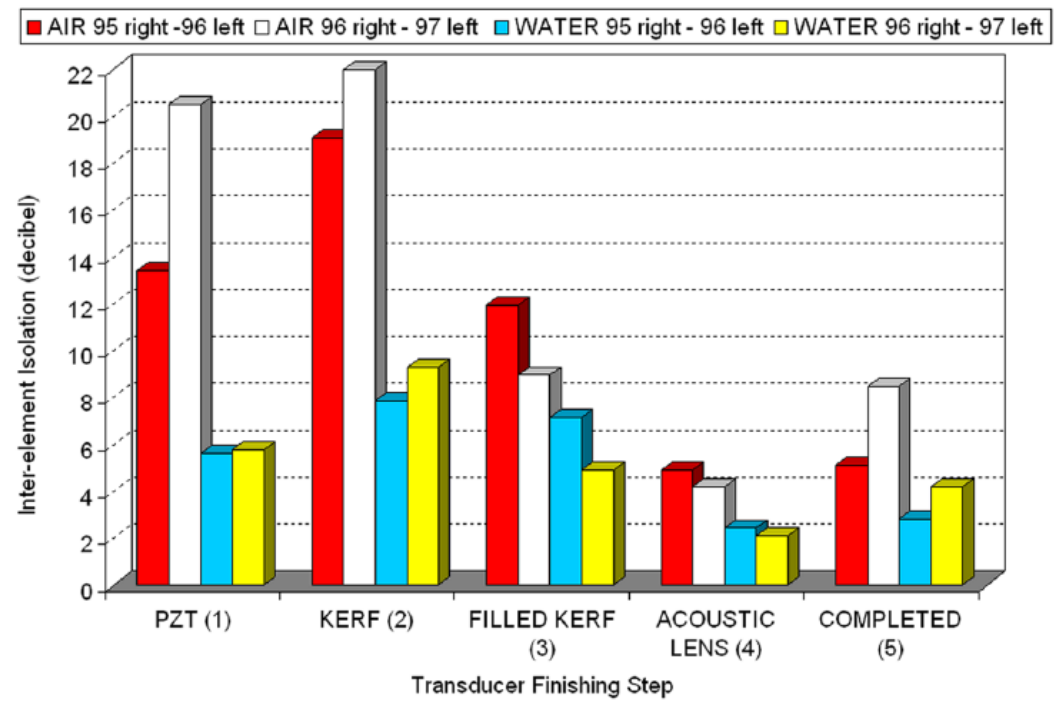

Figure 4. Inter-element isolation values.

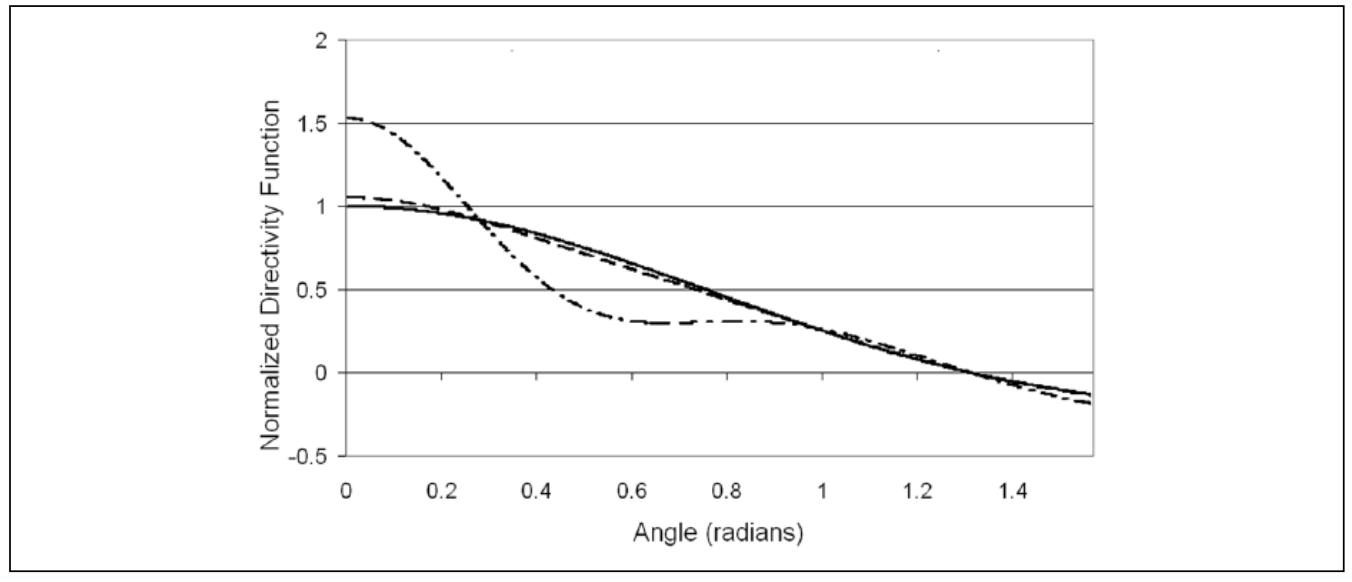

Figure 5. Directivity function for a single uncoupled element (solid) and for the same element having $-22 \mathrm{~dB}$ (dash) and $-2 \mathrm{~dB}$ (dash dot) inter-element isolation with the first neighboring one. All the functions are normalized to $F_{0}(0)$.

Figure 4 shows that the progression of the manufacturing process yields increasing cross-talk in air when the transducer with kerf (Step 2) is turned into a transducer with filling material and the matching layer (Step 3). At Step 4, when the transducer is also equipped with the acoustic lens, there is a further sensible decrease in the inter-element isolation due to the strong mechanical coupling enacted by the lens itself, whereas slight improvement of the probe completed with the socket is visible, and it is due to the inductive tuning (Step 5) that optimized the power transfer between the pulse generator and the probe by matching their impedances. Measurements under water documented an expectably poor inter-element isolation because the water increases both electrical and mechanical coupling. Finally, the comparison among the different finishing 


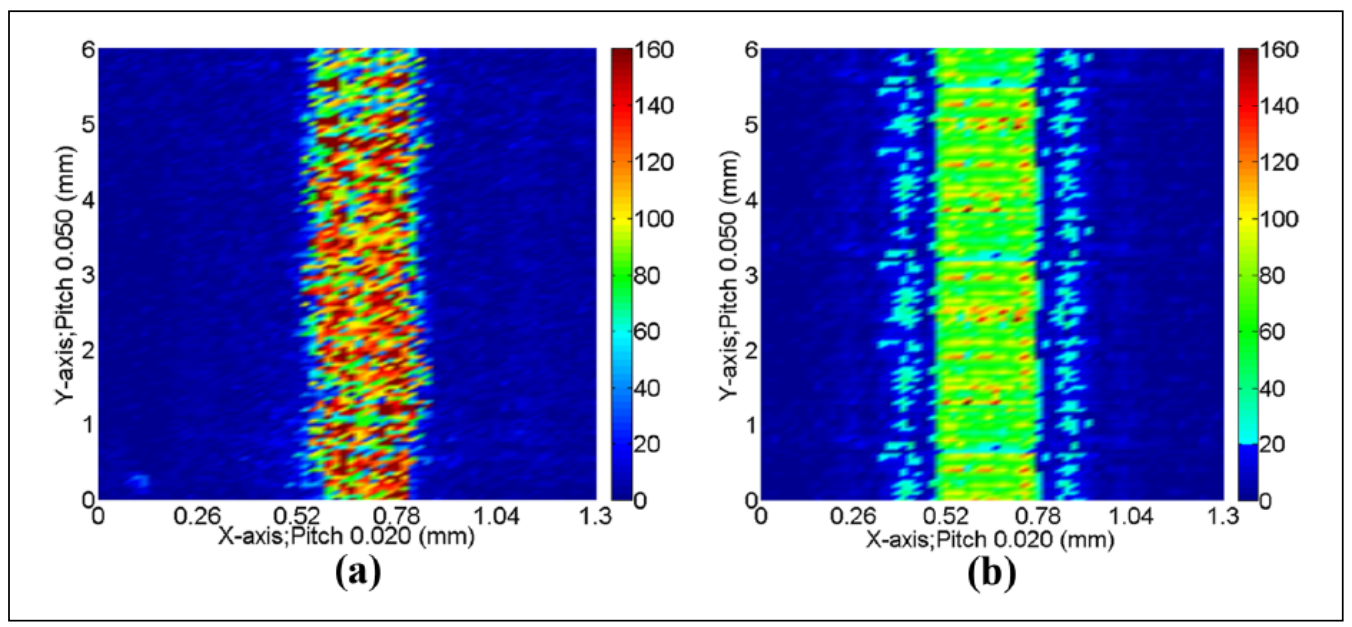

Figure 6. Vibration maps for the excited and neighboring elements in air: (a) with unfilled kerf and (b) with filled kerf. The color bars depict the displacement amplitude in nanometers.

stages in water showed a behavior very similar to that measured in air, that is, the isolation increases from Step 1 to 2, then decreases through Steps 2, 3, and 4, but more gradually with respect to air, and finally slightly increases from Step 4 to Step 5.

As expected, the major increase of inter-element isolation was measured in air for a transducer with unfilled kerf equal to $-22 \mathrm{~dB}$ (Step 2) and with an acoustic lens (Step 4) over $-4 \mathrm{~dB}$. Conversely, the poorest isolation was obtained with a transducer under water equal to $-9 \mathrm{~dB}$ (Step 2) and nearly $-2 \mathrm{~dB}$ (Step 4). The addition of cable, socket, and the inductive tuning (step 5) produced an overall increase of inter-element isolation of nearly $-8 \mathrm{~dB}$ and $-4 \mathrm{~dB}$ for measurements in air and water, respectively.

The vibration field was detected in a $1.3 \mathrm{~mm}$ span with a $20 \mu \mathrm{m}$ pitch along the $x$-axis, and a 6 $\mathrm{mm}$ span along the $y$-axis, with a $50 \mu \mathrm{m}$ pitch, both centered on the energized element. The total area was covered with approximately 8,000 points, corresponding to approximately $8 \mathrm{~mm}^{2}$. In Figures $6 \mathrm{a}$ and $6 \mathrm{~b}$, some typical vibration maps acquired in air are depicted at two different finishing stages relative to unfilled and filled kerfs. We can observe in Figure $6 \mathrm{~b}$ a second, smaller amplitude peak of mechanical cross-talk occurring in the first neighboring elements. The addition to the filler and the matching layers, it produced an overall increase in cross-talk of nearly $14 \mathrm{~dB}$.

Unlike the experimental set-up adopted in Felix et al. ${ }^{13}$ and Certon et al., ${ }^{14}$ the transducer surface has not been metalized to increase the reflectivity of the laser beam. Basically, we wanted to carry out tests that did not alter the main features of the array transducer and, in particular, to interfere as little as possible with the manufacturing process.

The LDV used by the investigators in Felix et al. ${ }^{13}$ and Certon et al. ${ }^{14}$ has a $100 \mathrm{~mW}$ output power with a $30 \mu \mathrm{m}$ diameter of the laser beam. As a result, the power density in the measurement area is approximately $14 \mathrm{~kW} \cdot \mathrm{cm}^{-2}$. However, the laser used in our work has a 0.8 to $1 \mathrm{~mW}$ output power with a $10 \mu \mathrm{m}$ spot diameter and a $175 \mathrm{~mm}$ focal distance, which corresponds to approximately $1.3 \mathrm{~kW} \cdot \mathrm{cm}^{-2}$. Because the macroscopic and microscopic damage, which a high output power concentration may cause to the transducer, is not a priori known, it seemed reasonable to limit the power density given the need for an acceptable signal-to-noise ratio during the tests.

The laser beam focus placed onto the array transducer surface is equal to waist size $\left(w_{0}\right)$. The radius $w(z)$ of the laser beam as a function of the probe surface distance $\Delta z$ from the focus is given by Svelto ${ }^{25}$ : 
Table I. Finishing Condition and Reflection Factor of the Reflecting Surfaces.

Surface Condition

Partially reflecting (metalized PZT)

Light matt (acoustic lens)

Black matt (filled kerf and matching layer)
Reflection Factor (\%)

From 60 to 70

From 50 to 60

From 3 to 5

$$
w(z)=w_{0} \cdot \sqrt{1+\left(\frac{\lambda_{0} \cdot \Delta z}{\pi \cdot w_{0}^{2}}\right)^{2}} .
$$

For a maximum change in the focusing distance $\Delta \mathrm{z}= \pm 5 \mathrm{~mm}$, the corresponding change in the laser beam of wavelength $\lambda_{0}$ diameter is $10 \mu \mathrm{m} \pm 33 \mu \mathrm{m}$. This also causes an increase in the spatial average effect when the vibration of a surface area of the transducers is measured because the nominal kerf width is $25 \mu \mathrm{m}$.

The resolving power actually depends on the finishing of the reflecting surfaces ${ }^{14}$ (Table 1). In our case, this yielded a minimum vibration amplitude spanning from approximately $0.6 \mathrm{~nm}$ for metalized surfaces (Step 1) to approximately $2 \mathrm{~nm}$ for black matt surfaces (Step 3). According to Certon, ${ }^{14}$ the displacement amplitude could be achieved with a standard deviation up to $10 \%$ for black matt surfaces.

\section{Electrical Impedance Measurements}

This measurement system is largely used and well-known because of its easy and fast data line testing. However, it does not seem possible to apply this technique to justify and analyze the experimental results obtained via laser vibrometry. A thorough interpretation of electrical impedance measurements needs a complete model of the electromechanical behavior of every transducer. Therefore, it is not possible to compare directly the impedance diagrams and the 1D models, such as Mason ${ }^{18}$ and Krimholtz et al. ${ }^{19}$

In Figure 7 we report the diagrams of the electrical impedance for a single piezoelement (n.96), which is measured via the spectrum/network analyzer HP4195A in air and under water.

The complexity of oscillating occurrences is particularly evident in the graph in Figure 8, which shows results for a transducer in air with kerf. From this diagram, it is possible to recognize the multiple resonances of a coupled oscillator system made of the series-connected PZT-1st matching-2nd matching. However, a detailed analysis of this well-known phenomenon is beyond the scope of the present work. Moreover, it is evident that with the introduction of the filler, the coupled vibrations tend to weaken because of the strong lateral constraint imposed by the filler itself. However, our results give evidence that a conspicuous amount of interacting phenomena should be kept in mind to minimize the energy spread among unwanted probe vibrations.

\section{Discussion and Conclusion}

Measurements of displacement amplitudes in commercialized arrays at various manufacturing stages produced by harmonic excitation have provided insight into inter-element coupling.

This has been documented by comparison with the oscillation amplitudes measured for the energized element and the neighboring ones. This analysis was performed on one transducer, which was the same at several stages of the manufacturing process, starting from the diced piezoelectric array to the complete acoustic stack (kerf, filler, matching layer, and finally, the acoustic 


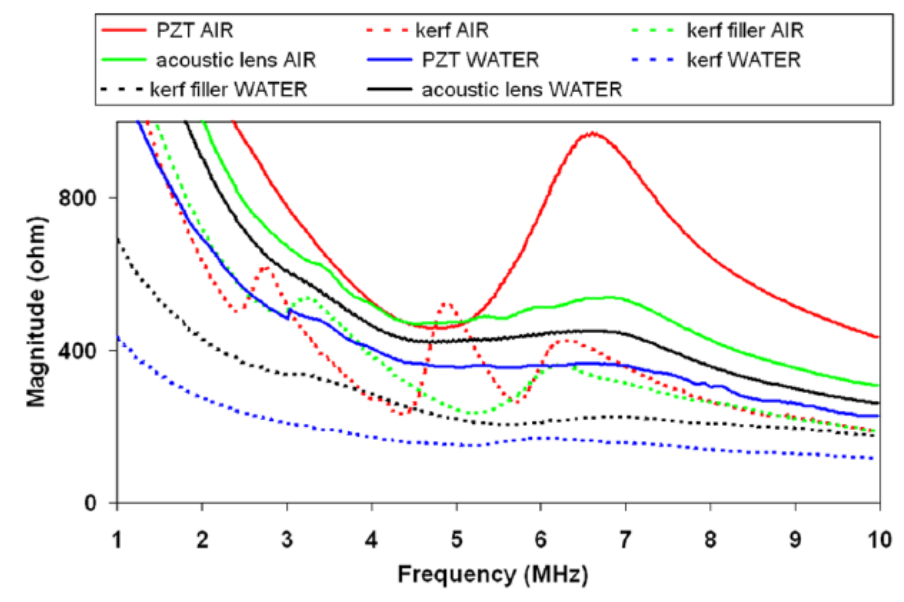

Figure 7. Impedance magnitude response of a single piezoelement (n.96, according to figure 2a).

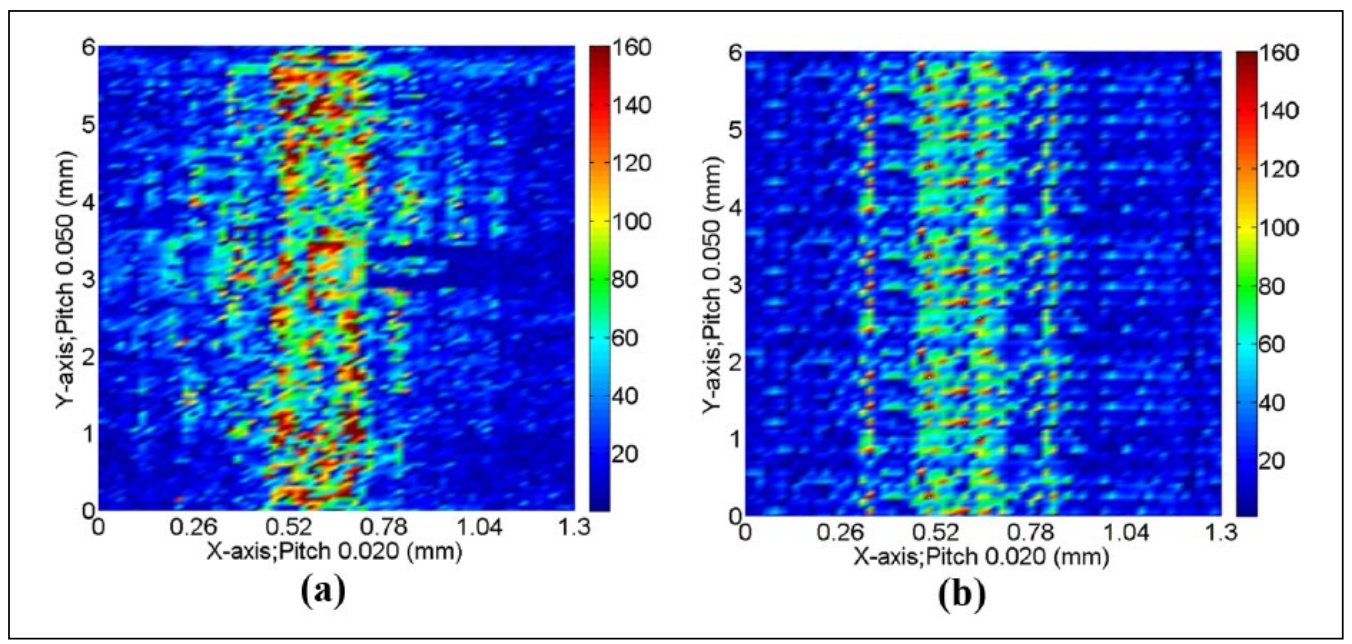

Figure 8. Vibration maps for the excited and neighboring elements in water: (a) with unfilled kerf and (b) with filled kerf. The color bars depict the displacement amplitude in nanometers.

lens). In a linear array transducer, the elements are separated by piezoelectrically inactive filling material, in particular epoxy resins ${ }^{20}$ whose stiffness value is somewhat unpredictable before the complete polymerization.

As recalled in Kino and DeSilets, ${ }^{2}$ the cross-coupling should be kept below $30 \mathrm{~dB}$ to achieve broad acceptance angle transducers. According to Kino and DeSilets, ${ }^{2}$ we found a major increase in the cross-coupling due to the introduction of the filling material that was over $14 \mathrm{~dB}$ in air and over $5 \mathrm{~dB}$ in water.

The sensitivity spanned from $(0.6 \pm 38 \%) \mathrm{nm} \cdot \mathrm{V}^{-1}$ to $(11 \pm 40 \%) \mathrm{nm} \cdot \mathrm{V}^{-1}$ when the energized element was tested in air and from $(0.6 \pm 57 \%) \mathrm{nm} \cdot \mathrm{V}^{-1}$ to $(8 \pm 67 \%) \mathrm{nm} \cdot \mathrm{V}^{-1}$ when the measurements were performed in water. The sensitivity of the neighboring elements spanned from 
$(0.4 \pm 48 \%) \mathrm{nm} \cdot \mathrm{V}^{-1}$ to $(1.8 \pm 42 \%) \mathrm{nm} \cdot \mathrm{V}^{-1}$ and from $(0.5 \pm 62 \%) \mathrm{nm} \cdot \mathrm{V}^{-1}$ to $(4 \pm 70 \%) \mathrm{nm} \cdot \mathrm{V}^{-1}$ in air and water, respectively.

Figure $3 \mathrm{~b}$ outlines the high standard deviation for the experimental data. Such results cannot be justified if the analysis is limited to the standard deviation of the measurement system, which is up to $10 \%$. The explanation for such high standard deviations can be found in two distinct phenomena: (a) acousto-optic effect arising in water and (b) the excitation of a Lamb wave on the transducer surface (both in air and in water). Previous works ${ }^{26-29}$ have shown that displacement LDV measurements in water are altered by acousto-optic interactions between the laser beam and the radiated pressure field. This justifies the higher standard deviation found in water with respect to measurements in air.

In Figures $7 \mathrm{a}$ and $7 \mathrm{~b}$, typical vibration maps acquired in water at two different finishing stages relative to unfilled and filled kerfs are shown. The effects of the acousto-optic interaction (Figures 7a and $7 \mathrm{~b}$ ) can be seen as non-zero ghost oscillation spots placed outside the excited and neighboring elements. For a more detailed estimation, according to Certon et al. ${ }^{27,28}$ and Sapozhnikov et al., ${ }^{29}$ we simulated the amount of this effect for the analyzed probes in the actual experimental conditions.

We have performed these tests on 10 transducers of three different types (i.e., 4 linear, 3 convex, and 3 phased array), and the final reproducibility of the measured oscillation amplitudes was computed up to (a) 13\% for convex arrays, (b) 10\% for linear arrays, and (c) $9 \%$ for phased arrays.

We found that the mean oscillation amplitude of the array elements was overestimated by 10 $\%$ maximum, whereas the standard deviation could be raised up to $30 \%$ with respect to that measured in air. These values can easily justify the increase in the standard deviation found in water measurements, but they do not add significant error to the average oscillation amplitudes.

A different motivation was found to interpret the standard deviation of measurements in air, which is probably due to the excitation and propagation of Lamb waves guided within the matching layers, both in air and in water (Figure $6 \mathrm{~b}$ and $7 \mathrm{~b}$ ). This vibration mode also appears in the oscillation pattern acquired in water (Figure $7 \mathrm{a}$ and $7 \mathrm{~b}$ ). In particular, the wavelength estimated for the first symmetrical $\left(\mathrm{S}_{0}\right)$ and anti-symmetrical $\left(\mathrm{A}_{0}\right)$ Lamb wave modes ${ }^{30,31}$ was roughly equal to $0.4 \mathrm{~mm}$ and $0.2 \mathrm{~mm}$, respectively. More specifically, the wavelength of $\mathrm{A}_{0}$ is in good agreement to that visible in Figures $6 \mathrm{~b}$ and $7 \mathrm{~b}$, which allows for the hypothesis of flexural oscillations launched within the matching layers. A detailed analysis of such parasitic modes is beyond the scope of the present work.

These preliminary experimental data offer quantitative information about the gradual and currently still unavoidable decrease of the inter-element isolation that accompanies manufacturing, together with some evidence of other phenomena related to the measurement technique (acoustooptic effect) or to the number and type of layers of the acoustical stack (excitation of Lamb waves).

As concluding remarks, we would like to underline that a satisfactory approach that predicts the behavior of array elements with backing, matching layers, filling material, and acoustic lens is still lacking. Moreover, it is difficult to simulate the behavior of a transducer array because its real acoustic properties depend on several unpredictable factors, such as the doping and/or the curing process. A balanced combination of computer simulations and experimental set-up will lead to design concepts that are more efficient for quality 32 control.

We believe that these results could be helpful to both researchers and manufacturing companies in the array design field.

\section{Acknowledgments}

The authors wish to thank Prof. Adriano Alippi and Prof. Andrea Bettucci for sharing their competence in setting up experiments, analyzing data, and for their permission to carry out the instrumentation at the Physical 
Acoustics Laboratory, "Sapienza" University of Rome. Furthermore, we deeply thank the Reviewers for their insightful comments, which improved the clarity of the manuscript.

\section{Declaration of Conflicting Interests}

The author(s) declared no potential conflicts of interest with respect to the research, authorship, and/or publication of this article.

\section{Funding}

The author(s) received no financial support for the research, authorship, and/or publication of this article.

\section{References}

1. Kino GS, Baer R. Theory for cross-coupling. IEEE Ultrason Symp. 1983;1:1013-9.

2. Kino GS, DeSilets CS. Design of slotted transducer arrays with matched backings. Ultrason Imaging. 1979;1189-209.

3. Dominguez IS, Contla PA, Hernandez EM, von Kruger MA. Crosstalk effects caused by the geometry of piezoelectric elements in matrix ultrasonic transducers. Braz J Biomed Eng. 2011;27:90-7.

4. Bybi A, Grondel S, Assaad J, Hladky-Hennion AC, Granger C, Rguiti M. Reducing crosstalk in array structures by controlling the excitation voltage of individual elements: a feasibility study. Ultrasonics. 2013;53:1135-40.

5. Bybi A, Grondel S, Assaad J, Hladky-Hennion AC, Granger C, Rguiti M. Extension of the crosstalk cancellation method in ultrasonic transducer arrays from the harmonic regime to the transient one. Ultrasonics. 2014;54:720-4.

6. Celmer M, Opielinski KJ. Study of crosstalk in linear ultrasonic transducer arrays. Acta Acust United Acust. 2015;101(1):46-54.

7. Guess JF, Oakley CG, Douglas SJ, Morgan RD. Cross-talk paths in array transducers. IEEE Ultrason Symp. 1995;2:1279-82.

8. Baer RL, Kino GS. Theory for cross coupling in ultrasonic transducer arrays. Appl Phys Lett. 1984;44:954-6.

9. Hanafy A. Vibration analysis of single element transducer in acoustic arrays. Ultrason Imaging. 1981;3(2):173-82.

10. Zhou SI, Wojcik GL, Hossack JA. An approach for reducing adjacent element crosstalk in ultrasound arrays. IEEE Trans Ultrason Ferroelectr Freq Control.2003;50(12):1752-61.

11. Desilets CS, Fraser JD, Kino GS. The design of efficient broad-band piezoelectric transducers. IEEE Trans Sonic Ultrason. 1978;25:115-25.

12. Lamberti N, Gori P, Iula A, Caliano G, Carotenuto R, Pappalardo M. Radiation pattern distorsion caused by interelement coupling in linear array. IEEE Ultrason Symp. 1999;2:1071-5.

13. Felix N, Certon D, Lacaze E, Lethiecq M, Patat F, Experimental investigation of cross-coupling and its influence on the elementary radiation pattern in 1D ultrasound arrays. IEEE Ultrason Symp. 1999:2:1053-6.

14. Certon D, Felix N, Lacaze E, Teston F, Patat F. Investigation of cross-coupling in 1-3 piezocomposite arrays. IEEE Trans Ultrason Ferroelectr Freq. 2001;48(1):85-92.

15. Dias JF. An experimental investigation of the cross-coupling between elements of an acoustic imaging array transducer. Ultrason Imaging. 1982;4:44-55.

16. Ayter S. Transmission line modelling for array transducer elements. IEEE Ultrason Symp. 1990;2: 791-4.

17. Lerch R. Simulation of piezoelectric devices by two and three dimensional finite elements. IEEE Trans Ultrason Ferroelectr Freq. 1990;37:233-47.

18. Mason WP. Electromechanical Transducers and Wave Filters. 2nd ed. Princeton, NJ: D. Van Nostrand Company Inc.; 1948.

19. Krimholtz R, Leedom DA, Matthaei GL. New equivalent circuits for elementary piezoelectric transducers. Electron Lett. 1970;6:398-9. 
20. Branca FP, Bini F, Marinozzi F, Grandoni A. Optimum choice of acoustic properties of filling materials using optical measurement. IEEE Trans Ultrason Ferroelectr Freq. 2004;3:1663-5.

21. Branca FP, Bini F, Grandoni A, Marinozzi F. Experimental investigation of spurious vibrations damping by different backings. In: Tilg B., Proceedings of the IASTED International Conference on Biomedical Engineering, February 16 - 18, 2004, Innsbruck, Austria, pp. 542-5.

22. Marinozzi F, Bini F, Biagioni A, Grandoni A, Spicci L. Note: comparative experimental studies on the performance of 2-2 piezocomposite for medical ultrasound transducers. Rev Sci Instrum. 2013;84(9):096110.

23. Drain LE. The Laser Doppler Technique. New York: John Wiley; 1980.

24. Dewhurst RJ, Shan Q. Optical remote measurement of ultrasound. Meas Sci Technol.1999;10:R139-68.

25. Svelto O. Principles of Lasers. New York: Plenum Press; 1989.

26. Royer D, Casula O. Quantitative imaging of transient acoustic fields by optical heterodyne interferometry. IEEE Ultrason Symp. 1994;2:1153-62.

27. Certon D, Bou Matar O, Guyonvarch J, Felix N, Patat F. Characterization of transducer arrays by laser interferometry: influence of acousto-optic interactions on displacement measurements in water. IEEE Ultrason Symp. 2001;2:1065-8.

28. Certon D, Ferin G, Bou Matar O, Guyonvarch J, Remenieras JF, Patat F. Influence of acousto-optic interactions on the determination of the diffracted field by an array obtained from displacement measurements. Ultrasonics. 2004;42:465-71.

29. Sapozhnikov OA, Morozov AV, Cathignol D. Acousto-optic interaction in laser vibrometry in a liquid. Acoust Phys. 2009;55:365-75.

30. Viktorov IA. Rayleigh and Lamb Waves. New York: Plenum Press; 1967.

31. Auld A. Acoustic Fields and Wave in Solids. New York, NY: John Wiley; 1992.

32. Marinozzi F, Branca FP, Bini F, Scorza A. Calibration procedure for performance evaluation of clinical Pulsed Doppler Systems. Measurement. 2012;45:1334-42. 